\title{
Isolated Tricuspid Valve Infective Endocarditis: A Rare Entity
}

\section{Serkan Burc Deser and Mustafa Kemal Demirag}

\author{
Department of Cardiovascular Surgery, Ondokuz Mayis University, Turkey
}

Corresponding author: Serkan Burc Deser, Medical Faculty, Department of Cardiovascular Surgery, Ondokuz Mayis University, Turkey, Tel: 903623121919; E-mail: sbd983@yahoo.com

Rec date: Aug 06, 2016; Acc date: Dec 23, 2016; Pub date: Dec 26, 2016

Citation: Deser SB, Demirag MK. Isolated Tricuspid Valve Infective Endocarditis: A Rare Entity. Med Case Rep. 2016, 2:4.

\section{Abstract}

Tricuspid valve (TV) infective endocarditis (IE) is an uncommon issue. Isolated TVIE accounts for approximately $5 \%$ to $36 \%$ of all IE. Staphylococcus aureus $(60 \%$ to $90 \%)$ is the most common microbiological pathogen. Most of the patients can be successfully treated with medical therapy however; surgery is preferred for patients in whom medical therapy has failed. Successful surgical treatment should include radical debridement of the infected tissues and the restoration of the valve function. We report the surgical management of a 32-year-old female who was presented with tricuspid valve endocarditis with unknown predisposing factors.

Keywords: Tricuspid valve endocarditis; Surgery; Treatment; Tricuspid valve regurgitation; Staphylococcus aureus

\section{Introduction}

Infective endocarditis (IE) often affects the left side of the heart however; the tricuspid valve (TV) is the most affected valve in the right heart chamber. Isolated TVIE accounts for approximately $5 \%$ to $36 \%$ of all IE. Mortality rate for left-sided IE is ranges from $20 \%$ to $30 \%$ however, right-sided IE ranges from $7 \%$ to $11 \%$. Staphylococcus aureus is the most common (60\% to $90 \%$ ) microbiological pathogen. Streptococci (20\%), Gram-negative bacilli (10\%), Coagulase-negative staphylococci, HACEK organisms (Haemophilus aphrophilus, Actinobacillus, Actinomycetes comitans, Cardiobacterium hominis, Eikenella corrodens and Kingella kingae), Enterococci spp., Gemella morbillorum, Pseudomonas aeruginosa, fungi and HIV are the other causes [1]. Predisposing factors are including alcoholism, immuno-depression, intravenous drug usage, cardiac implantable electronic devices, central venous catheters and congenital heart diseases. Fever, multiple pulmonary emboli and sustained bacteraemia can be the signs of right heart endocarditis. There may also be signs of right heart failure on physical examination. Medical therapy is the primary treatment strategy for isolated TV IE with less than $5 \%$ mortality rate. Most of the patients can be successfully treated with medical therapy however; surgery is preferred for patients in whom medical therapy has failed. Sepsis, septic emboli, right ventricular dilatation associated with severe tricuspid regurgitation and right heart failure, large vegetation $(>1 \mathrm{~cm})$, abscess formation and failure of antimicrobial therapy to control the infection are the main indications for surgery. Surgery reduces the long-term mortality [2]. Successful surgical treatment should include radical debridement of the infected tissues and restoration of the valve function. Time of surgery is still unclear. Several surgical modalities have been described. Surgical treatment options are including valve excision and valve replacement or valve reconstruction. Valvectomy without valve replacement has of historical interest which leads to systemic venous hypertension. Using cryopreserved mitral homograft or an upside down stent less aortic porcine valve experience is limited. We report the management of a 32-year-old female who was presented with tricuspid endocarditis with unknown predisposing factors.

\section{Case Presentation}

A 32-year-old female was referred to our hospital with prolonged high fever, arthralgia, 3 weeks duration of headache. On admission, her temperature was 40 centigrade celsius, blood pressure was $115 / 70 \mathrm{mmHg}$, pulse was 96 beats/min, respiratory rate was 24 , oxygen saturation while breathing room air was $96 \%$. Her physical examination revealed jugular veins distension with a hepatojugular reflux and a mild painful hepatomegaly. Laboratory tests revealed leukocytosis of $15.4 \mathrm{uL}$, anemia with hemoglobin of $10.2 \mathrm{~g} / \mathrm{dL}$, erythrocyte sedimentation rate of $76 \mathrm{~mm} / \mathrm{h}$ and C-reactive protein of $15.8 \mathrm{mg} / \mathrm{L}$. The patient had no history of cardiovascular disease, intravenous catheter placement or drugs abuse. Transthoracic echocardiography revealed large, mobile and pedunculated vegetation measuring $22 \times 16 \mathrm{~mm}$ which was attached to the anterior leaflet of the tricuspid valve with severe regurgitation and pulmonary hypertension (mean pulmonary arterial pressure was $37 \mathrm{mmHg}$ ). The right ventricle and atrium were enlarged. The left ventricle valves were normal. Staphylococcus aureus was isolated in blood cultures. Intravenous levofloxacin and gentamycin treatment was started. A control transesophageal echocardiography revealed enlargement of the vegetation in the tricuspid valve 1 week later $(6 \mathrm{~mm})$. We decided to perform valve surgery for persistent infection and enlarging vegetation despite adequate antibiotherapy. Routine preparation for surgery was made and informed consent was taken. Via cardiopulmonary bypass, 
large vegetation which was attached to the tricuspid valve was detected (Figure 1). The vegetation could not be removed with valve sparing. Tricuspid valve leaflets was resected and tricuspid valve was replaced with 33 no carbomedics bioprosthesis valve. The postoperative course was uneventful. Control transthoracic echocardiography revealed a mild tricuspid regurgitation without residual vegetation. Bacteriological analysis of the vegetation revealed the presence of neutrophils and organisms. Two weeks after the surgery most symptoms were ameliorated. Antibiotherapy was continued for 6 weeks and then the patient was discharged with clinical recovery. Control tranthoracic echocardiography was performed at 1 and 6 months after the surgery without any deterioration of the valve function. Six months later she was doing well with stable vital signs and no dyspnea or fever on follow up was detected.
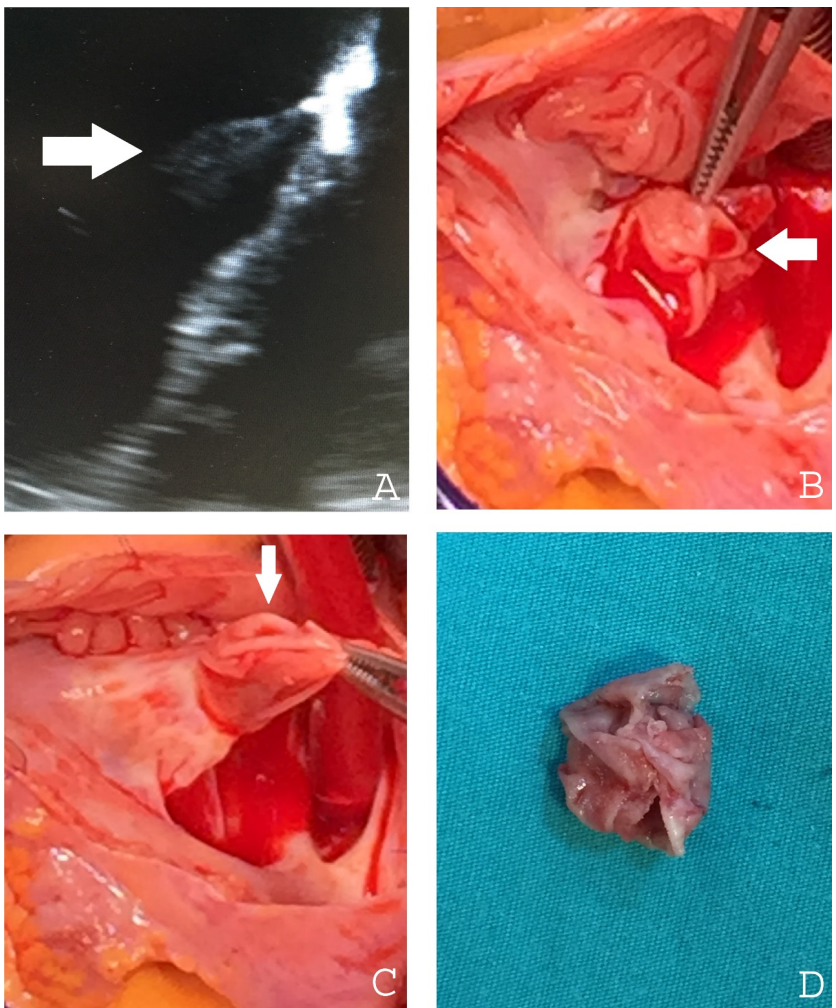

Figure $1 \mathrm{~A}$ transthoracic echocardiogram showing a vegetation (maximal length of $18 \mathrm{~mm}$ ) attached to the anterior leaflet of the tricuspid valve and no perivalvular extension of endocarditis (A), intra operative image of vegetation on the anterior leaflet showed by arrow $(B, C)$ and extracted tissue (D).

\section{Discussion}

Staphylococcus aureus or coagulase-negative Staphylococcus species are the common cause of right sided
IE. $70 \%$ to $85 \%$ of the right sided IE can be treated by medical therapy. Outcomes of combination of both medical and surgical treatment for fungal endocarditis are better than medical treatment alone. Indications for surgical therapy are including right heart failure secondary to the severe TV regurgitation, failure to appropriate antibiotherapy and diuretic therapy, large vegetations $(>10 \mathrm{~mm})$ and pulmonary emboli [3]. Early operation is recommended one week after the antibiotherapy was initiated. Tricuspid valve surgery has low morbidity, low recurrence rate and favorable long-term outcome [4]. Ring annuloplasty, vegetectomy, valve repairing with using an autologous pericardial patch, bicuspidalization valvuloplasty, neochorda with polytetrafluoroethylene and suture annuloplasty can be perfomed [5]. The successful treatment of tricuspid valve endocarditis should include the excision of all infected tissues and the restoration of the valve. If the repair is not technically feasible, valve replacement should be preferred. Biophrostetic valves have better durability in the low-pressure right-sided circulation. Pulmonary septic emboli are not a contraindication for surgery. Surgical treatment was indicated for our patient due to ongoing infection, enlarging vegetation and right heart failure despite taking appropriate antibiotherapy.

\section{Conclusion}

In conclusion, surgical treatment of tricuspid valve endocarditis remains controversial and vast majority of patients do not require surgical intervention for complete recovery.

\section{References}

1. Akinosoglou K, Apostolakis E, Koutsogiannis N, Leivaditis V, Gogos CA (2012) Right-sided infective endocarditis: surgical management. Eur J Cardiothorac Surg 42: 470-479.

2. Dawood MY, Cheema FH, Ghoreishi M, Foster NW, Villanueva RM (2015) Contemporary outcomes of operations for tricuspid valve infective endocarditis. Ann Thorac Surg 99: 539-546.

3. Shetty N, Nagpal D, Koivu S, Mrkobrada M (2016) Surgical and medical management of isolated tricuspid valve infective endocarditis in intravenous drug users. J Card Surg 31: 83-88.

4. Dawood MY, Cheema FH, Ghoreishi M, Foster NW, Villanueva RM, et al. (2015) Contemporary outcomes of operations for tricuspid valve infective endocarditis. Ann Thorac Surg 99: 539546.

5. Yong MS, Coffey S, Prendergast BD, Marasco SF, Zimmet AD, et al. (2016) Surgical management of tricuspid valve endocarditis in the current era: A review. International Journal of Cardiology 202: 44-48. 